\title{
Should we prove the balance of baseline data in randomized controlled trials?
}

\author{
Hyun Kang ${ }^{1}$ and Sangseok Lee ${ }^{2}$ \\ Department of Anesthesiology and Pain Medicine, ${ }^{1}$ Chung-Ang University College of Medicine, \\ ${ }^{2}$ Sanggye Paik Hospital, Inje University College of Medicine, Seoul, Korea
}

A table is often used as a means of presenting a specific outcome in medical research. The first table presented in the results section, (i.e., "Table 1"), generally includes demographic information and medical history, containing basic data like vital signs and measures. Researchers present the characteristics of patients included in the study (i.e., demographic, biological, medical baseline parameters). Additionally, in most studies, researchers often present a statistic ( $\mathrm{P}$ value) to indicate that differences in certain properties have minimal effects on outcomes. In particular, by showing that the $\mathrm{P}$ value did not reach a statistically significant level (i.e., $\mathrm{P}<0.05$ ) in randomized controlled trials, some researchers point out that patients' characteristics that could affect the outcome were well balanced among the groups.

However, $\mathrm{P}$ values presented to demonstrate that the baseline variables are balanced between groups do not provide sufficient evidence to reject the null hypothesis, rather which is a representative example that is not scientifically used.. Even if the randomization was properly accomplished, the significance of between-group differences in the baseline variables does not necessarily indicate clinical significance or relevance, but merely that the observed effect is attributable to chance. Indeed, statistically significant differences that can take place between baselines could occur accidentally after random assignment. However,

Corresponding author: Sangseok Lee, M.D.

Department of Anesthesiology and Pain Medicine, Sanggye Paik Hospital, Inje University College of Medicine, 1342 Dongil-ro, Nowon-gu, Seoul 01757, Korea

Tel: 82-2-950-1171, Fax: 82-2-950-1323

Email: s2248@paik.ac.kr

ORCID: https://orcid.org/0000-0001-7023-3668

Received: March 8, 2019.

Accepted: March 8, 2019.

Korean J Anesthesiol 2019 April 72(2): 89-90

https://doi.org/10.4097/kja.19093 few researchers seem to accept this fact without questioning it [1].

Despite the fact that it has long been known to be inappropriate to evaluate or present differences in baseline data of randomized controlled trials [2,3], research papers presenting $\mathrm{P}$ values for underlying variables are easily found. According to a study conducted in 1997, half of the randomized controlled trials $(50 \%)$ in leading journals reported on the significance of the baseline data [4]. Nevertheless, no medical journals published in Korea have been examined concerning this subject.

In this issue of the Korean Journal of Anesthesiology (KJA), Ahn et al. [5] evaluated a total of 312 randomized controlled trials published in the Journal of Anesthesiology between 2010 and 2017 analyzing Table 1 presented in each study. The authors concluded that, unlike the intentions of the researchers, it was not important to present $\mathrm{P}$ values in Table 1 as a means of showing that the random assignment has been carried out properly. Overall, 26.5\% (83/312) of the research papers published during this period presented a $\mathrm{P}$ value in Table 1 .

Since 2013, the KJA has announced an editorial policy recommending that no $\mathrm{P}$ values should be included in Table 1 of randomized controlled trials. As a result of this policy, $\mathrm{P}$ values appear to be present at a relatively lower rate than expected. However, the factual reality may be different. In this regard, only six of the papers presented a significant $\mathrm{P}$ value $(\mathrm{P}<0.05)$. Of the total 318 variables examined, only nine were reported to present statistically significant differences. This is likely to be statistically significant, and it is possible that this phenomenon,

\footnotetext{
1) The "invisible hand" describes the unintended social benefits of an individual's self-interested actions. Adam Smith first introduced the concept in The Theory of Moral Sentiments, written in 1759, invoking it in reference to income distribution. Although used here in a completely different meaning from the original text, the authors used the phrase "invisible hand" as a parody of linguistic play.
}

(c) This is an open-access article distributed under the terms of the Creative Commons Attribution Non-Commercial License (http://creativecommons.org/ licenses/by-nc/4.0/), which permits unrestricted non-commercial use, distribution, and reproduction in any medium, provided the original work is properly cited. 
in which the variables of significance in Table 1 are less reported, may have not been caused by chance, or "the invisible hand"1) may have been operating. In other words, the researcher did not report the baseline variable that resulted in significant differences after randomization, did not report the total $\mathrm{P}$ value in the study, or recreated the patient for the baseline variable with significant differences. In addition, it is possible that the reviewer may have doubted the appropriateness of the study's random assignment if there were significant differences in the basic variables during the process of reviewing the research.

Considering baseline data similarity to the extent that it is possible to overcome this possibility is suggested as one of the cases where bias after the random assignment is suspected [6]. Randomization is essential to reduce the likelihood of selection and confounding bias that may occur when randomly assigning subjects in a randomized study. However, understanding of randomized research still seems to be lacking. Adequate knowledge of randomization can help researchers, reviewers, and readers have a profound understanding and insight into research and assist in establishing the research plan correctly. In addition, whether or not to evaluate or suggest differences in baseline data when conducting and reporting randomized controlled trials should also be considered.

\section{Conflicts of Interest}

Dr. Kang and Dr. Lee are the boarding members of Statistical Rounds of Korean Journal of Anesthesiology. The authors declare no conflicts of interest.

\section{ORCID}

Hyun Kang, https://orcid.org/0000-0003-2844-5880

Sangseok Lee, https://orcid.org/0000-0001-7023-3668

\section{References}

1. Lee S, Kang H. Statistical and methodological considerations for reporting RCTs in medical literature. Korean J Anesthesiol 2015; 68: 10615.

2. Roberts C, Torgerson DJ. Understanding controlled trials: baseline imbalance in randomised controlled trials. BMJ 1999; $319: 185$.

3. Senn S. Testing for baseline balance in clinical trials. Stat Med 1994; 13: 1715-26.

4. Assmann SF, Pocock SJ, Enos LE, Kasten LE. Subgroup analysis and other (mis)uses of baseline data in clinical trials. Lancet 2000; 355 : 1064-9.

5. Ahn EJ, Kim JH, Kim TK, Park JH, Lee DK, Lee S, et al. Assessment of P values for demographic data in randomized controlled trials. Korean J Anesthesiol 2019; 72: 130-4.

6. Page M. RoB 2.0: A revised tool to assess risk of bias in randomized trials [Internet]. London: Cochrane [cited 2019 March 8]. Available from https://training.cochrane.org/resource/rob-20-webinar. 